\title{
Antiarrhythmic management and implantable defibrillator use in survivors of prehospital cardiac arrest without myocardial infarction in West Yorkshire
}

\author{
C B Pepper, P D Batin, M Ryder, J Bannister, J C Cowan, A F Mackintosh
}

\begin{abstract}
Objective-To explore the current use of secondary preventive treatment in survivors of out of hospital cardiac arrest without myocardial infarction (primary ventricular tachycardia/ventricular fibrillation (VT/VF)) in West Yorkshire, and assess the implications of recent studies on the benefits of implantable cardioverter-defibrillators (AICD) in this context.

Design-Retrospective analysis of an ambulance service based database of outcome after resuscitation of out of hospital cardiac arrest and the Leeds AICD implantation database.

Main outcome measures-Mortality, rate of referral for specialist investigation, antiarrhythmic treatment.

Results-Twelve month mortality following successful discharge after primary VF arrest was $15 \%$. Of 53 patients with primary VF/VT, 29 apparently did not see a cardiologist during the initial admission. Amiodarone was the most widely used antiarrhythmic agent. Six patients (15\%) received an AICD. During the same period 22 patients from the same catchment area received an AICD following an in-hospital cardiac arrest.

Conclusions-Mortality among survivors of non-infarct related prehospital cardiac arrest remains significant, with few patients being referred for specialist investigation. The implementation of recent guidelines on AICD use in cardiac arrest survivors would have resulted in an approximate $60 \%$ increase in the total numbers of defibrillators implanted in the West Yorkshire area.
\end{abstract}

(Heart 2000;83:312-315)

Keywords: ventricular fibrillation; ventricular tachycardia; primary arrhythmias; implantable cardioverter-defibrillator

Yorkshire Heart

Centre, Leeds General Infirmary, Great George St, Leeds LS1 3EX, West Yorkshire, UK

C B Pepper

J C Cowan

Pinderfields Hospital, Wakefield, West Yorkshire, UK

P D Batin

Department of Cardiology, St James Hospital, Leeds, West Yorkshire, UK

M Ryder

J Bannister

A F Mackintosh

Correspondence to: Dr Pepper

Accepted 9 September 1999
The increasing availability of ambulance staff trained in defibrillation has resulted in significant early survival from out of hospital cardiac arrest. ${ }^{1-3}$ Patients surviving cardiac arrest without acute myocardial infarction remain at high risk of recurrent malignant arrhythmias and require careful investigation and treatment. Despite this, the rate of referral for specialist management in the United Kingdom has been low. ${ }^{4}$

Since 1987 we have prospectively identified and followed up out of hospital cardiac arrests in West Yorkshire which involved paramedics or ambulance crews equipped with a defibrillator. In the present study we investigate recent practice regarding the use of antiarrhythmic drugs and referral for specialist electrophysiological assessment in survivors of out of hospital arrest without myocardial infarction. We attempted to assess the implications of recent evidence ${ }^{67}$ on the management of these patients.

\section{Methods}

Patients suffering out of hospital cardiac arrest were identified from West Yorkshire Ambulance Service (WYMAS) return forms as previously described. ${ }^{1}$ Further information was obtained from accident and emergency (A\&E) department and inpatient records and supplemented from general practitioners as necessary. The present paper contains data on arrests occurring between January 1987 and September 1997 Data were not recorded for a 10 month period during 1989 to 1990 owing to industrial action. Acute myocardial infarction was diagnosed on the basis of the appearance of fresh electrocardiographic $\mathrm{Q}$ waves or left bundle branch block, plus increase in serum creatine kinase or other cardiac enzyme levels to twice the upper range of normal for that hospital.

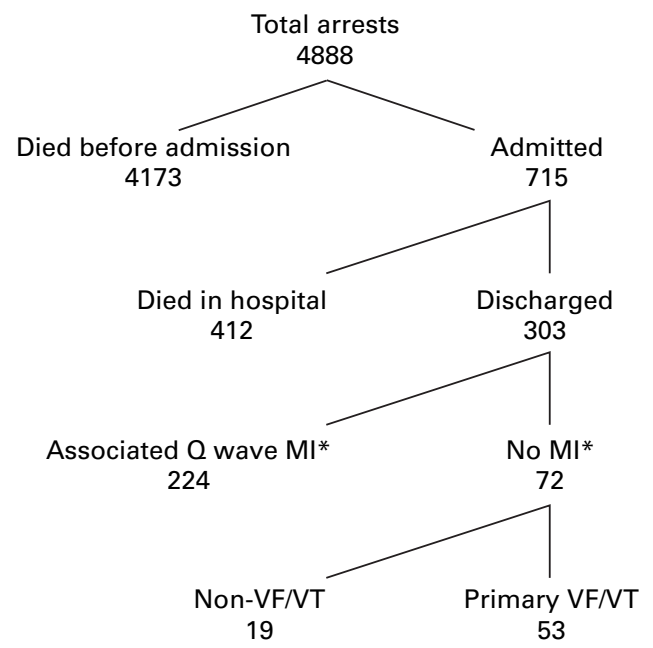

Figure 1 Outcome of cardiac arrests attended by West Yorkshire paramedic ambulance crews, Fanuary 1987 to September 1997. ${ }^{\star}$ Classification of myocardial infarction was not possible in seven patients owing to inadequate information. 
Table 1 Primary VT/VF: patient characteristics and outcome

\begin{tabular}{lc}
\hline & $n(\%)$ \\
\hline $\begin{array}{l}\text { Median age (years) (range) } \\
\text { Sex }\end{array}$ & $68(28$ to99) \\
Male & $33(63)$ \\
Female & $20(37)$ \\
Presenting hospital & \\
District general hospital & $36(68)$ \\
University hospital & $17(32)$ \\
CPS at discharge & $47(89)$ \\
I & $4(8)$ \\
II & $2(4)$ \\
III & $28(53)$ \\
Antiarrhythmic treatment at discharge & $1(1.9)$ \\
Amiodarone & $5(9.4)$ \\
Sotalol & $2(3.8)$ \\
Other $\beta$ blocker & $6(11.3)$ \\
Class I agents & $5(9.4)$ \\
AICD & $6(11.3)$ \\
Nil & \\
Not stated & $13(25)$ \\
Other investigations/treatment & $1(1.9)$ \\
Coronary angiography & $2(3.8)$ \\
PTCA & $8(15)$ \\
CABG & \\
EP study & \\
\hline AICD, automated implantable cardioverter-defibrillator; \\
CABG, coronary artery bypass graft; CPS, cerebral perform- \\
ance score; EP, electrophysiology; PTCA, percutaneous translu- \\
minal coronary angioplasty. & \\
&
\end{tabular}

All patients were prospectively registered with the Office of National Statistics (ONS) for identification of deaths, and death drafts were obtained in all cases. In addition general practitioners were approached on an annual basis to confirm patient survival.

\section{Results}

Between January 1987 and September 1997, details were recorded of 4888 patients suffering a prehospital cardiac arrest and attempted resuscitation by West Yorkshire ambulance staff. Of these, $303(6 \%)$ survived to hospital discharge (fig 1). Seventy two patients were discharged with a diagnosis of arrest unrelated to acute myocardial infarction. In $57(80 \%)$, the first recorded rhythm was ventricular fibrillation or tachycardia. Four cases of ventricular fibrillation/ventricular tachycardia (VF/VT) had apparent reversible underlying pathology (arising out of bradycardia owing to complete heart block or sick sinus syndrome in two, and secondary to hypoxia in two).

PRIMARY VF/VT

The characteristics and management of the 53 patients experiencing VT/VF in the absence of any reversible precipitating event (for the purposes of this paper, primary VT/VF) are detailed in table 1 . Thirty seven per cent were female and $63 \%$ male. Median follow up was 38.5 months (range 4-129), 46 patients having completed at least 12 months of follow up. The overall and neurological status of these survivors at discharge was generally good, 96\% reported to have a Glasgow-Pittsburgh overall and cerebral performance score (OPS and CPS, table 2) of I or II (that is, capable of independent living). All cause mortality at one year was seven of $46(15 \%)$ and 20 of $53(38 \%)$ had died by the end of follow up. The certified cause of death was cardiac in the majority of cases (six of seven at one year and 14 of 20 at the end of follow up). It was not possible to reliably ascertain the proportion of patients experiencing "sudden" cardiac death; however, three had a death certificate diagnosis of cardiac arrest and seven of myocardial infarction.

Two thirds of the patients initially presented to one of seven district general hospitals in the WYMAS area (table 1), all of which had a cardiologist. The remaining patients were seen at one of two university centres, one of which contained the regional electrophysiology unit. The chance of there being documented involvement of a cardiologist was higher in a university hospital ( 15 of $17,88 \%$ ), than in a district general hospital (14 of 36, 39\%). The rate of coronary angiography and revascularisation was low and showed no evidence of increasing throughout the time course of the study.

Some form of antiarrhythmic prophylaxis was reported in 42 of 53 patients. Fifty three per cent received amiodarone, prescribed in all but two cases without electrophysiological study; $\beta$ blockers were used in only $11 \%$ of this population of patients known to have a high prevalence of ischaemic heart disease. Two patients received empirically prescribed class I antiarrhythmic agents (one mexilitene and one disopyramide). In 11 patients there was no evidence of antiarrhythmic prophylaxis (including $\beta$ blockade) on discharge.

Eight patients (15\%) are known to have been referred for detailed electrophysiological assessment. Two were discharged on maintenance amiodarone treatment, and AICD implantation was intended in six. One patient receiving an AICD also received long term amiodarone treatment. In one patient AICD implantation was technically unsuccessful owing to an unacceptably high defibrillation threshold, and the patient was subsequently referred for cardiac transplantation. Two of the five patients ultimately receiving an AICD had experienced appropriate defibrillator therapy during the follow up period. Four of the five patients remained alive at the time of analysis.

Table 2 Glasgow-Pittsburgh cerebral and overall performance categories

\begin{tabular}{llll}
\hline Cerebral performance & Overall performance \\
\hline I & $\begin{array}{l}\text { Good cerebral performance. Conscious. Alert. Able to } \\
\text { work and lead a normal life }\end{array}$ & I & $\begin{array}{l}\text { Good overall performance. Healthy, alert, capable of } \\
\text { normal life }\end{array}$ \\
II & $\begin{array}{l}\text { Moderate cerebral disability. Conscious. Sufficient } \\
\text { cerebral function for part time work in sheltered } \\
\text { environment or independent activities of daily life }\end{array}$ & II & $\begin{array}{l}\text { Moderate overall disability. Conscious. Moderate cerebral } \\
\text { disability alone (CPC II) or moderate disability from } \\
\text { non-cerebral dysfunction alone or both }\end{array}$ \\
III & $\begin{array}{l}\text { Severe cerebral disability. Conscious. Dependent on others } \\
\text { for daily support because of impaired brain function }\end{array}$ & III & $\begin{array}{l}\text { Severe overall disability. Conscious. Dependent on others } \\
\text { for daily support }\end{array}$ \\
IV & $\begin{array}{l}\text { Coma. Vegetative state } \\
\text { Dead }\end{array}$ & V & $\begin{array}{l}\text { Coma. Vegetative state } \\
\text { Dead }\end{array}$ \\
V & &
\end{tabular}


AICD IMPLANTS

The Leeds General Infirmary provides a supraregional service for AICD implantation. Of the 85 AICD systems implanted during the study period, 63 were in patients from the West Yorkshire ambulance catchment area. Forty nine West Yorkshire patients with AICD systems remained alive by the end of 1997 (all cause mortality $12.3 \%$ ). Thirty two of these devices were implanted for cardiac arrest, 22 occurring in hospital and 10 out of hospital.

\section{Discussion}

In this study we report the use of antiarrhythmic treatment, including AICD, in survivors of non-infarct-related out of hospital cardiac arrest in West Yorkshire between 1987 and 1997. Despite the poor natural history of this condition and the availability of potentially effective treatment, the rate of referral for specialist assessment was low, with only $55 \%$ of patients seeing a cardiologist during the index admission (although some may have been referred for subsequent assessment), and eight of 53 patients being referred for further electrophysiological assessment. There was no evidence of changing referral patterns over the period of the study in the present population, despite a steady increase in overall AICD implantation rates in Leeds, which predominantly reflected the advent of prepectoral units and their increased ease and safety of implantation. A low rate of referral among survivors of prehospital cardiac arrest has also been found in previous studies. ${ }^{458}$ While this may be appropriate when VF is secondary to acute myocardial infarction, it does not seem appropriate in a group of patients without acute ischaemia who are known to have a significant risk of recurrence.

The low rate of referral for detailed assessment may be partly explained by a high prevalence of concurrent illness, including neurological damage; however, this seems unlikely to reflect a large proportion, as $96 \%$ of patients were capable of independent living on discharge. In contrast to this, 22 patients in the West Yorkshire area received an AICD after in-hospital cardiac arrest over the same time period. Although the proportion of survivors of in-hospital arrest that this represents is unknown, it raises the possibility that hospital doctors treat in-hospital arrests more actively than prehospital arrest, although there would be little or no clinical justification for such an approach.

Historical mortality data preceding the widespread use of antiarrhythmic prophylaxis suggests a two year all cause mortality in this population as high as $45 \% .{ }^{9}$ More recent data suggest a better prognosis, with mortality of the order of $10-15 \%$ in aggressively treated patients. ${ }^{10}{ }^{11}$ Mortality in the present series is consistent with this, and similar to that in the pharmacologically treated arm of the antiarrhythmics versus implantable defibrillators (AVID) study. ${ }^{6}$ Despite these apparently improving figures, this remains a group at high risk of adverse outcome in which aggressive intervention should be considered.
Evidence based antiarrhythmic drug use in this population is difficult. The majority of early data involved widespread use of VaughanWilliams class I antiarrhythmic agents, now known to be associated with significant proarrhythmia, ${ }^{12}$ and perhaps unsurprisingly a significant rate of recurrence remained despite suppression of inducible arrhythmia at electrophysiological study. In this context Moosvi et $a l$, in a retrospective study of 209 survivors of cardiac arrest, showed a probable adverse effect of empirical, predominantly class I, antiarrhythmic prescription. ${ }^{13}$ The CASCADE study (cardiac arrest in Seattle: conventional versus amiodarone drug evaluation), published in 1993, suggested survivors of cardiac arrest fared better with empirical use of amiodarone than with electrophysiology guided treatment, again with predominantly class I agents. ${ }^{11}$ There was no major change in the use of amiodarone $(12 / 20 v 6 / 32)$ or in referrals for electrophysiological study $(2 / 20 v 6 / 32)$ before or after the study was published. There are no studies of a direct comparison of amiodarone $v$ placebo in that population. The experience of the European myocardial infarct amiodarone trial (EMIAT) ${ }^{14}$ and the Canadian amiodarone myocardial infarction arrhythmia trial (CAMIAT) $^{15}$ (both published towards the end of the period covered by our study) suggests that amiodarone is unlikely to be actively harmful, although benefit after cardiac arrest remains unproven.

The development of the implantable cardioverter-defibrillator has provided an alternative option for the prevention of sudden death in patients with malignant ventricular arrhythmias. Recent studies have established a reduction in cardiac mortality from AICD treatment over conventional treatment, suggesting a 30\% reduction in mortality compared to best available drug therapy. ${ }^{6}$ Such data have led to the American Heart Association and American College of Cardiology recommendation that defibrillator therapy should be the treatment of choice in survivors of sudden cardiac death not associated with a clear reversible cause. ${ }^{7}$ Applying these guidelines to the present population would result in a significant increase in defibrillator implant rates. Using arbitrary criteria for eligibility for AICD (age not more than 75 years and class I cerebral performance score after the arrest), 39 extra devices would have been implanted over the 10 year study period, increasing the West Yorkshire implant rate by $61 \%$. Strict application of the AVID criteria would result in a considerably higher implant rate even than this, as only around $50 \%$ of the AVID population had suffered cardiac arrest. Indeed, estimates derived from coronary care unit admissions elsewhere in the United Kingdom have suggested that application of AVID criteria to this population would result in up to a sevenfold increase in AICD implant rates. ${ }^{16}$

In conclusion, the rate of referral for specialist assessment among survivors of primary VF/VT causing prehospital cardiac arrest in West Yorkshire between 1987 and 1997 was low, a situation which probably reflected 
practice throughout the United Kingdom. In the light of current evidence, many more patients should be considered for specialist referral with a view to AICD treatment. This would lower the significant mortality still associated with this condition.

1 Wright D, Bannister J, Ryder M, et al. Resuscitation of patients with cardiac arrest by ambulance staff with extended training in West Yorkshire. BMF 1990;301:600-2 2 Cobbe SM, Redmond MJ, Watson JM, et al. "Heartstart of hospital defibrillation. BMF 1991;302:1517-20.

3 Lombardi G, Gallagher J, Gennis P. Outcome of out-ofhospital cardiac arrest in New York City. The pre-hospital arrest survival evaluation (PHASE) study. FAMA 1994; 271:678-83.

4 Dalziel K, Carrington DJ, Cobbe SM. Clinical profile and management of patients discharged alive after pre-hospital defibrillation [abstract]. Eur Heart f. 1992;13 (suppl):293

5 Weston CFM, Avery PG, Stephens MR. Management of survivors of pre-hospital ventricular defibrillation. $f R R$ Coll Physicians Lond 1993;27:242-6.

6 The Antiarrhythmics Versus Implantable Defibrillators (AVID) Investigators. A comparison of antiarrhythmicdrug therapy with implantable defibrillators in patients resuscitated from near fatal ventricular arrhythmias. N Engl f Med 1997;337:1576-83.

7 Gregoratos G, Cheitlin MD, Conill A, et al. ACC/AHA guidelines for implantation of cardiac pacemakers an antiarrhythmia devices: a report of the ACC/AHA Task Force on Practice Guidelines (Committee on Pacemaker Implantation). F Am Coll Cardiol 1998;31:1175-206.
8 Batin PD, Bannister J, Ryder M, et al. Management and Batin PD, Bannister J, Ryder $\mathrm{M}$, et al. Management and diac arrest. $\mathcal{F} R$ Soc Physicians Lond 1997;31:280-6.

9 Baum RS, Alvarez H, Cobb LA. Survival after resuscitation from out-of-hospital ventricular fibrillation. Circulation 1974;50:1231-5.

10 Myerburg RJ, Kessler KM, Estes D, et al. Long-term survival after prehospital cardiac arrest: Analysis of outcome during an 8 year study. Circulation 1984;70:53846.

11 The CASCADE Investigators. Randomized antiarrhythmic drug therapy in survivors of cardiac arrest (the CASCADE study). Am 7 Cardiol 1993;72:280-7.

12 The Cardiac Arrhythmia Suppression Trial (CAST) Investigators. Preliminary report: effect of encainide and flecainide on mortality in a randomised trial of arrhythmia suppression after myocardial infarction. $N$ Engl $\mathcal{F} \mathrm{Med}$ 1989;321:406-12.

13 Moosvi AR, Goldstein S, Medendorp SV, et al. Effect of empiric antiarrhythmic drug therapy in resuscitated out-of-hospital cardiac arrest victims with coronary artery disease. Am f Cardiol 1990;65:1192-7.

14 Julian DG, Camm AJ, Frangin G, et al. Randomised trial of effect of amiodarone on mortality in patients with left ventricular dysfunction after recent myocardial infarction: EMIAT. Lancet 1997;349:667-74.

15 Cairns JA, Connolly SJ, Roberts R, et al. Randomised trial of outcome after myocardial infarction in patients with frequent or repetitive ventricular premature frequent or repetitive ventricular prem
depolarisations: CAMIAT. Lancet 1997;349:675-82.

16 Pathmanathan RK, Lau EW, Cooper J, et al. Potential impact of antiarrhythmic drugs versus implantable defibrillators on the management of ventricular arrhythmias: the Midlands trial of empirical amiodarone versus electrophysiologically guided intervention and cardioverter implant registry data. Heart 1998;80:68-70. 